\title{
Automação no laboratório de microbiologia
}

\author{
Juliane de Mello Fonseca1, Inneke Marie van der Heijden ${ }^{1}$
}

${ }^{1}$ Disciplina de Microbiologia e Imunologia, Departamento de Patologia, Centro Universitário Saúde ABC (FMABC) - Santo André (SP), Brasil - E-mail: inneke.heijden @ fmabc.br

DOI: https://dx.doi.org/10.7322/abcshs.v44i2.1313

Nesse fascículo da ABCS Health Science foi publicado um artigo de Rodrigues et al. ${ }^{1}$ comparando as metodologias fenotípicas manual e automatizada para a identificação de microrganismos isolados de hemocultura. A pesquisa foi realizada a partir de estudo observacional e retrospectivo de amostras provenientes do laboratório de microbiologia clínica de um Hospital Universitário do Rio Grande do Norte e teve como objetivo avaliar o impacto da automação versus identificação manual frente ao tratamento, internação e mortalidade dos pacientes da Unidade de Terapia Intensiva (UTI). Embora os autores deste estudo não tenham observado vantagens significativas da automação em relação aos desfechos clínicos analisados, em um estudo publicado por Doern et al. ${ }^{2}$ foi observado que o fornecimento precoce dos resultados de identificação e suscetibilidade de microrganismos isolados a partir de diferentes culturas proporciona um manejo mais eficiente dos doentes e está associado a significativos benefícios clínicos e financeiros, uma vez que permite uma redução das taxas de mortalidade e dos custos gerais de hospitalização.

A rotina nos exames de microbiologia clínica é baseada no cultivo e isolamento de colônias puras dos microrganismos, identificação fenotípica e testes de sensibilidade aos antimicrobianos utilizados para o tratamento. A automação aplicada para identificação de microrganismos emprega testes fenotípicos que exploram as diferenças metabólicas dos diferentes gêneros e espécies de bactérias e fungos de importância epidemiológica ${ }^{3}$. Estes sistemas automatizados têm sido cada vez mais utilizados na rotina dos exames realizados nos laboratórios de microbiologia clínica, principalmente naqueles que processam um grande volume de amostras diariamente, visto que podem aprimorar o fluxo de trabalho, diminuir o tempo e os custos, além de permitir uma maior precisão na identificação dos microrganismos ${ }^{3,4}$. De acordo com estudo publicado por Donay et al. ${ }^{5}$, os sistemas automatizados podem proporcionar uma série de vantagens na rotina do laboratório de microbiologia tais como aumento de produtividade, diminuição no tempo de obtenção do resultado das identificações microbianas, aprimoramento no controle de qualidade com aumento de reprodutibilidade e precisão dos resultados e até mesmo um aumento na capacidade de rastrear e analisar os resultados microbiológicos.
Desde o lançamento da automação AutoMicrobic System, projetado no final dos anos 1960 pela McDonnell Douglas a pedido da NASA, uma infinidade de produtos tem surgido no mercado mundial ${ }^{4}$. Os principais equipamentos atualmente disponíveis neste campo são: o sistema automatizado Phoenix (lançado pela Becton Dickinson em 2003), o sistema MicroScan WalkAway (fabricado por Dade Behring INC., USA), o sistema Vitek (introduzido pela bioMérieux em 1997) e o sistema MALDITOFMS (Matrix Associated Laser Desorption-Ionization - Time of Flight - Mass Spectrometry) que se baseia na técnica de espectrometria de massa (MS) para identificar microrganismos ${ }^{6,7}$. Estudos comparativos entre estas metodologias têm sido descritos na literatura mundial ${ }^{8-12}$.

Em laboratórios de análises clínicas, quando estes possuem uma alta demanda de amostras que serão submetidas ao setor de microbiologia, estas tecnologias podem ser aplicadas, principalmente para diagnóstico de infecções hospitalares ou como metodologia padrão em laboratórios de referência, uma vez que a necessidade da automação faz-se indispensável para dar suporte à procura, apesar do custo inicial ser alto. A automação se mostra consideravelmente vantajosa neste setor das análises clínicas, proporcionando uma maior agilidade na obtenção dos resultados finais e melhor precisão na identificação de diversas espécies de micro-organismos, os quais são aspectos importantes na rotina do laboratório clínico e microbiológico, minimizando o tempo para a realização de diagnósticos convencionais e otimizando, por exemplo, a terapia antimicrobiana.

É importante salientar que os métodos automatizados contribuem muito para a otimização de um exame microbiológico, embora não seja possível às vezes mensurar seu impacto clínico por conta da dificuldade de realização destes estudos clínico-laboratoriais. De qualquer forma, vale ressaltar que ao acelerarmos os resultados microbiológicos, como isolamento e identificação dos principais microrganismos epidemiologicamente relevantes, estamos proporcionando ao médico assistente as escolhas mais apropriadas de antibioticoterapia. Assim, os pacientes podem reagir mais rápido e ter alta em menos tempo, minimizando sua permanência no hospital e assim diminuindo a emergência de infecções hospitalares, especialmente por bactérias mais resistentes. 


\section{REFERÊNCIAS}

1. Rodrigues CAO, Araújo GM, Silveira IA, Martins RR. Automated phenotypic method versus manual method in the identification of microorganisms isolated from blood cultures: clinical and microbiological outcomes. ABCS Health Sci. 2019;44(2):96-102. https://doi.org/10.7322/abcshs.v44i2.1156

2. Doern GV, Vautour R, Gaudet M, Levy B. Clinical impact of rapid in vitro susceptibility testing and bacterial identification. J Clin Microbiol. 1994;32(7):1757-62.

3. Burnham CA, Dunne WM, Greub G, Novak SM, Patel R. Automation in the clinical microbiology laboratory. Clin Chem. 2013;59(12):1696-702.

http://dx.doi.org/10.1373/clinchem.2012.201038

4. Chatzigeorgiou KS, Sergentanis TN, Tsiodras S, Hamodrakas SJ, Bagos PG. Phoenix 100 versus Vitek 2 in the identification of gram-positive and gram-negative bacteria: a comprehensive metaanalysis. J Clin Microbiol. 2011;49(9):3284-91. http://dx.doi.org/10.1128/JCM.00182-11

5. Donay JL, Mathieu D, Fernandes P, Prégermain C, Bruel P, Wargnier $\mathrm{A}$, et al. Evaluation of the automated phoenix system for potential routine use in the clinical microbiology laboratory. J Clin Microbiol. 2004;42(4):1542-6.

https://doi.org/10.1128/jcm.42.4.1542-1546.2004

6. Anhalt JP, Fenselau C. Identification of bacteria using mass spectrometry. Anal Chem. 1975;47(2):219-25. https://doi.org/10.1021/ac60352a007

7. Pasternak J. Novas metodologias de identificação de microorganismos: MALDI-TOF. Einstein (São Paulo). 2012;10(1):118-9. http://dx.doi.org/10.1590/S1679-45082012000100026
8. Kulah C, Aktas E, Comert F, Ozlu N, Akyar I, Ankarali H. Detecting imipenem resistance in Acinetobacter baumannii by automated systems (BD Phoenix, Microscan WalkAway, Vitek 2); high error rates with Microscan WalkAway. BMC Infect Dis. 2009;16(9):30.

http://dx.doi.org/10.1186/1471-2334-9-30

9. Pfennigwerth N, Kaminski A, Korte-Berwanger M, Pfeifer Y, Simon $\mathrm{M}$, Werner $\mathrm{G}$, et al. Evaluation of six commercial products for colistin susceptibility testing in Enterobacterales. Clin Microbiol Infect. 2019; ahead of print. https://doi.org/10.1016/j.cmi.2019.03.017

10. Woodford N, Eastaway AT, Ford M, Leanord A, Keane C, Quayle RM, et al. Comparison of BD Phoenix, Vitek 2, and MicroScan automated systems for detection and inference of mechanisms responsible for carbapenem resistance in Enterobacteriaceae. J Clin Microbiol. 2010;48(8):2999-3002. http://dx.doi.org/10.1128/JCM.00341-10

11. Fernández-Cuenca F, Tomás M, Tormo N, Gimeno C, Bou G, Pascual A. Reporting identification of Acinetobacter spp genomic species: A nationwide proficiency study in Spain. Enferm Infecc Microbiol Clin. 2019;37(2):89-92.

http://dx.doi.org/10.1016/j.eimc.2018.02.004

12. Jin WY, Jang SJ, Lee MJ, Park G, Kim MJ, Kook JK, et al. Evaluation of VITEK 2, MicroScan, and Phoenix for identification of clinical isolates and reference strains. Diagn Microbiol Infect Dis. 2011:70(4):442-7.

http://dx.doi.org/10.1016/j.diagmicrobio.2011.04.013 